
allemande

51-1 | 2019

La République démocratique allemande dans l'espace public européen (1949-2018)

\title{
Die DDR und die EWG 1957-1990
}

\section{Maximilian Graf}

\section{(2) OpenEdition \\ Journals}

Édition électronique

URL : https://journals.openedition.org/allemagne/1352

DOI : 10.4000/allemagne.1352

ISSN : 2605-7913

Éditeur

Société d'études allemandes

Édition imprimée

Date de publication : 2 juillet 2019

Pagination : 21-35

ISSN : 0035-0974

Référence électronique

Maximilian Graf, "Die DDR und die EWG 1957-1990“, Revue d'Allemagne et des pays de langue allemande [Online], 51-1 | 2019, Online erschienen am: 02 Juli 2020, abgerufen am 01 Juni 2022. URL: http:// journals.openedition.org/allemagne/1352 ; DOI: https://doi.org/10.4000/allemagne.1352 


\section{Die DDR und die EWG 1957-1990*}

\section{- Maximilian Graf**}

\section{Einleitung}

Der Aufsatz untersucht den Umgang der DDR mit der Europäischen Wirtschaftsgemeinschaft (EWG) von 1957 bis 1990. Zeitgenössisch wurde die Thematik anhand der rechtlichen Rahmenbedingungen und vor allem im Kontext der deutsch-deutschen Beziehungen sowie des - bis zum Ende der DDR in der Bundesrepublik so bezeichneten - innerdeutschen Handels problematisiert ${ }^{(1)}$. Die historische Forschung hat die Haltung des SED-Regimes zur europäischen Integration bisher primär als Perzeptionsgeschichte abgehandelt und deren Bedeutung für die ostdeutsche Wirtschaft kaum thematisiert ${ }^{(2)}$. Im Mittelpunkt der folgenden Analyse stehen daher die ökonomische und die politische Dimension des Verhältnisses der EWG zur DDR. Nach einer problemorientierten Einführung in die zentralen deutsch-deutschen und europäischen Rahmenbedingungen wird die vermeintliche Sonderstellung der DDR im Verhältnis zum Gemeinsamen Markt hinterfragt und die bisher von der

* Les travaux qui ont conduit à cet article s'inscrivent dans le projet PanEur1970s, qui a bénéficié du soutien du Conseil européen de la recherche (ERC) dans le cadre du programme de recherche et d'innovation de l'Union européenne Horizon 2020 (convention de subvention n 669194).

** Wissenschaftlicher Mitarbeiter am Europäischen Hochschulinstitut in Florenz.

1 Beispielsweise: Peter Scharpf, Europäische Wirtschaftsgemeinschaft und Deutsche Demokratische Republik, Tübingen, Mohr, 1973; Claus-Dieter EHLERMAnN, „Innerdeutsche Wirtschaftsbeziehungen und Europäische Gemeinschaft“, in: Ders., Siegfried Kupper, Horst Lambrecht, Gerhard Ollig (Hg.), Handelspartner DDR - Innerdeutsche Wirtschaftsbeziehungen, Baden-Baden, Nomos, 1975, S. 205-260; Bernhard JANSEN, EWG und DDR nach Abschluß des Grundlagenvertrages, BadenBaden, Nomos, 1977; Reinhold Biskup, Deutschlands offene Handelsgrenze. Die DDR als Nutznießer des EWG-Protokolls über den innerdeutschen Handel, Berlin, Ullstein, 1976.

2 Jana WüstenH AGEN, „Blick durch den Vorhang“: Die SBZ/DDR und die Integration Westeuropas (19461972), Baden-Baden, Nomos, 2001; Werner Müller, „Ex oriente luxus? - Supranationale Verflechtung der DDR in der Sicht des SED-Politbüros“, Jahrbuch für historische Kommunismusforschung, Nr. 14 (2000/2001), S. 187-212; Klaus-Peter ScнміD T, Die Europäische Gemeinschaft aus Sicht der DDR (1957-1989), Hamburg, Verlag Dr. Kovac, 1995 (2. Aufl.). 
Forschung kaum thematisierten Auswirkungen der fortschreitenden westeuropäischen Integration auf den ostdeutschen Staat in den Blick genommen. Trotz der ökonomischen Folgen behielt die DDR gemäß der von der Sowjetunion vorgegebenen Linie des Rats für Gegenseitige Wirtschaftshilfe (RGW) ihre strikte Nichtanerkennungspolitik gegenüber der EWG bei ${ }^{(3)}$. Dies änderte sich erst als Moskau Mitte der 1980er-Jahre unter Michail Gorbatschow seinen Standpunkt änderte. Daraufhin verdichteten sich die Kontakte zwischen Ost-Berlin und Brüssel und es folgte eine kurze Phase offizieller Beziehungen. Diese werden im letzten Abschnitt erstmals auf Basis kürzlich freigegebener DDR-Archivquellen dargestellt und bieten neue Einblicke in die europäische Dimension des beginnenden deutschen Einigungsprozesses im Herbst des Jahres 1989.

\section{Deutsch-deutsche und europäische Rahmenbedingungen}

Auf welcher Basis stand das Verhältnis der EWG zur DDR vor dem Hintergrund der deutschen Teilung? Um die Grundlagen zu verstehen, muss man in die Zeit vor der Gründung der EWG zurückgehen und die ökonomische Dimension der deutschen Zweistaatlichkeit in den Blick nehmen: Der innerdeutsche Handel zwischen der Bundesrepublik Deutschland und der DDR wurde auf Basis der Abkommen über den Interzonenhandel aus den Jahren 1949 und 1951 abgewickelt und galt als Binnenhandel. Dies wurde bereits bei der Gründung der Europäischen Gemeinschaft für Kohle und Stahl (EGKS) 1952 von allen Mitgliedsstaaten anerkannt; Bonn war nur verpflichtet, über den Warenfluss zu berichten. Anlässlich der Gründung der EWG 1957 konnte die Bundesrepublik ein Zusatzprotokoll zu den Römischen Verträgen durchsetzen, wonach der innerdeutsche Handel von diesen unberührt bleibe. Damit war auch das westdeutsche Wiedervereinigungsgebot in die DNA der entstehenden Gemeinschaft eingebrannt. War die DDR dadurch ohne eigenes Zutun zu einem „heimlichen Mitglied“ der EWG geworden? Drei Jahrzehnte wurde der aus dem innerdeutschen Handel resultierende Status der DDR in Ost und West kontrovers diskutiert. Jüngere Forschungen haben die Frage nach dem „heimlichen Mitglied“ aber zu Recht tendenziell mit nein beantwortet. Die DDR war zwar durch den innerdeutschen Handel privilegiert, darüber hinaus hatte sie aber kaum Vorteile. Westdeutsche Politiker sahen sich in Brüssel dennoch wiederholt dem Vorwurf ausgesetzt, dass die DDR ein „heimliches“ oder „13. Mitglied“ zum Vorteil der Bundesrepublik sei. Vor allem Frankreich und die Niederlande, aber auch Großbritannien thematisierten die potentielle Beeinträchtigung des Gemeinsamen Markts. Viel Berechtigung hatten die Vorwürfe nicht, da der Anteil der in den EWG-Markt re-exportierten Importe aus der DDR marginal war ${ }^{(4)}$.

3 Eine ausführliche Darstellung dazu soll unter dem Titel „Nichtanerkennung zu eigenen Lasten? Die DDR und die EWG in den ,langen 1970er-Jahren“" im Jahrbuch für historische Kommunismusforschung 2020 erscheinen.

4 Detlef NAKAтH, „Die DDR - ,heimliches Mitglied“ der Europäischen Gemeinschaft? Zur Entwicklung des innerdeutschen Handels vor dem Hintergrund der westeuropäischen Integration“, in: Franz Knipping, Matthias Schönwald (Hg.), Aufbruch zum Europa der zweiten Generation. Die europäische Einigung 1969-1984, Trier, WVT Wissenschaftlicher Verlag, 2004, S. 451-473. 
Blickt man auf die jeweiligen Handelsvolumen wird klar, warum auch der aus gesamtvolkswirtschaftlicher Sicht vernachlässigbare, privilegierte westdeutsche Zugang zum DDR-Markt in der EWG ein ständiges Politikum darstellte. Frankreich war in der Regel der größte Handelspartner der DDR aus dem EWG-Raum, das bilaterale Handelsvolumen überstieg aber kaum jemals das Ausmaß von zehn Prozent des innerdeutschen Handels. Die westdeutsche Dominanz auf dem als vielversprechend angesehenen DDRMarkt stand daher im Zentrum der Pariser Kritik ${ }^{(5)}$.

Die Vorwürfe der sozialistischen „Bruderstaaten“ an die DDR, die sich mit ihrer Haltung im RGW zeitweise an den Rande der Isolation brachte ${ }^{(6)}$, hatten aus ökonomischer Perspektive mehr Berechtigung, da die DDR durch den innerdeutschen Handel eine Vielzahl an Vorteilen im Zugang zum bundesdeutschen und somit zum gewichtigsten Teil des sich entwickelnden Gemeinsamen westeuropäischen Markts hatte. Wie sahen die in Ost und West kritisch beäugten Privilegien der DDR konkret aus?

1) Der innerdeutsche Handel galt als Binnenhandel. Er wurde zollfrei und später auch mit begünstigter Mehrwertsteuer abgewickelt. Damit fielen für andere Staaten bestehende finanzielle Belastungen im Handel mit der Bundesrepublik für die DDR weg.

2) Der innerdeutsche Handel wurde auf Basis von Verrechnungseinheiten abgewickelt (im Verhältnis 1 Verrechnungseinheit $=1$ Deutsche Mark $=1$ Mark der DDR). Ost-Berlin benötigte also für in diesem Rahmen abgewickelte Importe aus der Bundesrepublik keine Devisen. Wenn man bedenkt, dass die Bundesrepublik nicht nur für die DDR der wichtigste westliche Wirtschaftspartner war, sondern dies sukzessive auch für die anderen sozialistischen Staaten wurde, kann man sich die Dimension dieses Vorteils vorstellen.

3) Der Handel wurde im Clearingverfahren abgewickelt. In sich wandelnder Form und Volumen stand ein technischer Kredit, der sogenannte Swing, bereit. Gedacht als gegenseitig auszugleichende Konten eines bilanzierten Handels wurde der Swing von der DDR über weite Strecken ihrer Existenz als ein in großer Höhe auszuschöpfender zinsloser Kredit verwendet. Derartige Kredite standen anderen sozialistischen Staaten nicht zur Verfügung(7).

Die Vorteile der DDR gewannen an Bedeutung, als die Bundesrepublik ab Mitte der 1960er-Jahre auch im Verhältnis zur DDR die ökonomischen Vorläufer der „neuen Ostpolitik“ umsetzte. Nachdem Bonn in den 1950er-Jahren und insbesondere 1960 mit der temporären Kündigung des Abkommens über den Interzonenhandel

5 Ulrich PfeIl, Die „anderen“ deutsch-französischen Beziehungen. Die DDR und Frankreich 1949-1990, Köln/Weimar/Wien, Böhlau, 2004, S.600; Christian Wenkel, Auf der Suche nach einem „anderen Deutschland“. Das Verhältnis Frankreichs zur DDR im Spannungsfeld von Perzeption und Diplomatie, München, De Gruyter Oldenbourg, 2014, S. 163-167.

6 Jana Wüstenhagen, „RGW und EWG. Die DDR zwischen Ost- und Westintegration“, in: Ulrich Pfeil (Hg.), Die DDR und der Westen. Transnationale Beziehungen 1949-1989, Berlin, Links, 2001, S. 135-149, hier S. 139.

7 Zum innerdeutschen Handel siehe: Peter E. Fässler, Durch den „Eisernen Vorhang“. Die deutschdeutschen Wirtschaftsbeziehungen 1949-1969, Köln, Böhlau, 2006; Michael Kruse, Politik und deutsch-deutsche Wirtschaftsbeziehungen von 1945 bis 1989, Berlin, Verlag Dr. Köster, 2005; Peter Krewer, Geschäfte mit dem Klassenfeind. Die DDR im innerdeutschen Handel 1949-1989, Trier, Kliomedia, 2008. 
durchaus eine konfrontative Strategie versucht hatte, wurde nach dem Mauerbau dazu übergegangen, Ost-Berlin Zugeständnisse abzukaufen. Die Rahmenbedingungen des innerdeutschen Handels wurden weiter verbessert. Der Swing wurde erhöht und als dynamischer Swing auf maximal 25\% des Handelsvolumens festgesetzt. Zudem stellte die Bundesrepublik sicher, dass der 1968 implementierte gemeinsame EWG-Außenzoll keine Rückwirkungen auf die DDR hatte. Vor diesem Hintergrund verwundert es kaum, dass die nach staatlicher Anerkennung lechzende SED-Führung in den Verhandlungen über den Grundlagenvertrag 1972 rasch von der Idee eines staatlichen Handelsabkommens zwischen der Bundesrepublik und der DDR Abstand nahm. Die ökonomischen Vorteile des innerdeutschen Handels überwogen politische Erwägungen ${ }^{(8)}$. Die Bundesrepublik setzte dies unter ihren EWG-Partnern ebenfalls durch - freilich primär aus politischen Motiven. Entscheidend war das Argument, dass der innerdeutsche Handel einer der wenigen Aspekte sei, die der Verfestigung der deutschen Teilung entgegenstehen würden. Dies entsprach auch der grundlegenden Linie der sich entwickelnden EWG-Ostpolitik gegenüber den sozialistischen Staaten ${ }^{(9)}$.

Nicht zuletzt aus politischen Gründen lehnte die DDR grundsätzlich jede Form von westeuropäischer Integration ab, ganz gleich, ob es sich um den Marshall-Plan, die EGKS, die Europäische Verteidigungsgemeinschaft oder schließlich die EWG handelte. Einmal galt die europäische Integration als Projekt des „US-Imperialismus“ (der „ökonomische Arm der NATO“), dann wieder als auf die Unterwerfung des Kontinents abzielende Strategie des „westdeutschen Imperialismus“. Konsequent wurde das baldige Scheitern der EWG prognostiziert, da eine kapitalistische Integrationsform zwangsläufig an ihren inneren Widersprüchen zerbrechen müsse. In den internen Analysen setzte sich aber langsam ein größerer Realismus durch. Trotz Festhalten an überideologisierter Sprache und dementsprechenden Schlussfolgerungen dürfte spätestens Mitte der 1960er-Jahre zumindest der Ministerialbürokratie im Außenhandels- und Außenpolitikbereich klar gewesen sein, dass die EWG keinesfalls auf dem Weg in den unvermeidlichen Untergang war ${ }^{(10)}$. Auf höchster politischer Ebene fand man sich Ende der 1960er-Jahre mit der EWG als Realität ab, gleichzeitig wollte man sie aber nicht als politischen Faktor respektieren. Sie wurde „als politische und wirtschaftliche Gefahr“ angesehen und implizit anerkannte man ihre „wirtschaftliche Effizienz“, „politische Attraktivität“ und „Sogwirkung“. Dem Problem sollte aber in abgestimmter Weise durch die Mitglieder des RGW begegnet werden ${ }^{(11)}$.

8 Detlef Naкатн, Deutsch-deutsche Grundlagen. Zur Geschichte der politischen und wirtschaftlichen Beziehungen zwischen der DDR und der Bundesrepublik in den Jahren 1969 bis 1982, Schkeuditz, Schkeuditzer Buchverlag, 2002, S.322-360.

9 Angela Romano, „Untying Cold War knots: The EEC and Eastern Europe in the long 1970s“, Cold War History, 14/2 (2014), S. 153-174.

10 J. Wüstenhagen, „Blick durch den Vorhang“ (Anm. 2); K.-P. Schmidt, Die Europäische Gemeinschaft aus Sicht der DDR (Anm. 2).

11 W. Müller, „Ex oriente luxus?“(Anm. 2), S. 201. 


\section{Auswirkungen der westeuropäischen Integration auf die DDR}

Auf ihrem Haager Gipfel im Dezember 1969 verständigten sich die EWG-Mitglieder auf eine ambitionierte Agenda der Erweiterung und Vertiefung der Integration. Der erste Erweiterungsschritt folgte 1973 mit den Beitritten Großbritanniens, Irlands und Dänemarks, 1981 wurde Griechenland aufgenommen, 1986 folgten Spanien und Portugal. Auf die RGW-Staaten wirkten sich zunächst vor allem die im Rahmen der Vertiefung erhöhten Kompetenzen der Gemeinschaft in den Bereichen Außenpolitik und Außenhandel direkt aus. Während die handelspolitischen Implikationen trotz aller Rückschläge und Verzögerungen unbestreitbar sind, lässt sich über die im Rahmen der Europäischen Politischen Zusammenarbeit (EPZ) tatsächlich erreichten Fortschritte streiten. Jedoch machte die entstehende EPZ rasch klar, dass die Europäische Wirtschaftsgemeinschaft (EWG) keine die sowjetische Dominanz über Osteuropa stärkenden Abkommen schließen würde. Dies war eine eindeutige Absage an ein von der Sowjetunion angestrebtes Handelsabkommen zwischen der EWG und dem RGW. Genau darüber gab es im RGW aber massive Konflikte, da Staaten wie Rumänien, Polen und Ungarn eine flexiblere Politik und bilaterale Arrangements mit der EWG anstrebten $^{(12)}$. Die DDR folgte hingegen der sowjetischen Linie, nicht nur aus Loyalität, sondern eben auch weil sie weniger betroffen war $^{(13)}$.

Nicht nur im RGW spielten nationalstaatliche Interessen eine wichtige Rolle, sondern auch in der EWG. Diese und vor allem die Wirtschaftskrise der frühen 1970er-Jahre (mit dem Ende von Bretton Woods, dem ersten Ölschock und steigenden Arbeitslosenzahlen) verzögerten die Übertragung der alleinigen Außenhandelskompetenz an die EWG bis 1973, mit einer Übergangsfrist bis 1975 für bestehende Handelsabkommen. Fortan waren keine bilateralen Abkommen mehr möglich und alle Verträge waren mit der EWG-Kommission auszuhandeln. Dies galt nach langwieriger Abstimmung innerhalb der EWG ab Oktober 1974 auch für die DDR, die von allen Mitgliedstaaten nun als Drittstaat behandelt wurde. Dem bundesdeutschen Alleinvertretungsanspruch folgend hatte bis 1972/73 kein EWG-Staat die DDR diplomatisch anerkannt und demzufolge waren auch keine staatlichen Handelsabkommen geschlossen worden. Die Wirtschaftsbeziehungen intensivierten sich aber seit Ende der 1960er-Jahre und nach der diplomatischen Anerkennung wurden EWG-konforme bilaterale wirtschaftliche Rahmenverträge mit allen Mitgliedsstaaten unterzeichnet. Diese wurden in Ost-Berlin als Fortschritt gewertet und waren auch im Interesse der jeweiligen EWG-Staaten, die in der DDR einen vielversprechenden Markt für ihre Produkte und Technologien sahen ${ }^{(14)}$.

Die aus der sich vertiefenden westeuropäischen Integration resultierenden ökonomischen Probleme der sozialistischen Staaten blieben in den 1970er-Jahren bestehen

12 Suvi Kansikas, Socialist Countries Face the European Community: Soviet-bloc Controversies over East-West Trade, Brüssel, Peter Lang, 2014; Dies., „Room to manoeuvre? National interests and coalition-building in the CMEA, 1969-74“, in: Sari Autio-Sarasmo, Katalin Miklóssy (Hg.), Reassessing Cold War Europe, London, Routledge, 2011, S. 193-209.

13 Sitzung des SED-Politbüros am 6.6.1972, Stiftung Archiv der Parteien und Massenorganisationen der ehemaligen DDR im Bundesarchiv, Berlin (SAPMO-BArch), DY 30/J IV 2/2A/1601, Bl. 1-36.

14 Sitzung des SED-Politbüros am 17.12.1974, SAPMO-BArch, DY 30/J IV 2/2A/1845, Bl. 8, 54-67. 
und machten Kompromisse nötig. Gewisse Kontakte der RGW-Mitgliedsstaaten zu den EWG-Institutionen waren unvermeidlich. Um die Nichtanerkennung der EWG formal aufrechtzuerhalten wurden diese als „technische Kontakte“ qualifiziert. Sie ermöglichten einigen RGW-Staaten ihre Exporte in den Gemeinsamen Markt in geregelter und einvernehmlicher Form fortzusetzen. Die EWG sicherte sich gegen PreisDumping ab, im Gegenzug wurden die RGW-Exporteure von zusätzlichen Kosten befreit. Die DDR lehnte diese „technischen Kontakte“ ab, musste ihre Haltung aber bereits 1975 aufgeben. Nachdem Frankreich und Italien begonnen hatten, zusätzliche Gebühren auf DDR-Exporte von Schweinefleisch und Lebendschweinen einzuheben, brachen diese ein, und das für die Exportplanerfüllung verantwortliche Außenhandelsministerium empfahl der SED-Führung Anfang 1975 umgehend und mit Erfolg, ebenfalls „technische Kontakte“ zur EWG-Kommission aufzunehmen ${ }^{(15)}$.

Bald zeigte sich, dass in der Praxis weder Moskau noch Ost-Berlin ihr Dogma der Forderung nach Beziehungen zwischen EWG und RGW sowie die Ablehnung direkter Verhandlungen mit der Wirtschaftsgemeinschaft aufrechterhalten konnten. Als die EWG im Januar 1977 ihre gemeinsame Fischereipolitik zu implementieren begann, forderte sie die interessierten RGW-Staaten Polen, die Sowjetunion und die DDR dazu auf, die Größe ihrer Fischfangflotte bekanntzugeben und in Verhandlungen über Fangquoten einzutreten oder andernfalls die Fangtätigkeit innerhalb der EWGGewässer einzustellen. Trotz grundsätzlicher Ablehnung engagierte sich die DDR in mehreren Gesprächsrunden, ehe die Verhandlungen schlussendlich an der EWG-Forderung nach der Aufnahme der Berlin-Klausel in das Abkommen scheiterten. Wenig überraschend wurde die Bundesrepublik für die verhärtete EWG-Verhandlungsposition verantwortlich gemacht ${ }^{(16)}$.

In der Forschung besteht keine Einigkeit darüber, wie sehr das westliche integrierte Europa seine gemeinsame Außenhandelspolitik gegenüber den sozialistischen Staaten durch diverse bilaterale Abkommen selbst konterkarierte. Die Importe in den Gemeinsamen Markt waren jedenfalls effektiv und tatsächlich vergemeinschaftet. Dennoch blieben viele wirtschaftspolitische Aspekte weiterhin in der Kompetenz der Nationalstaaten (Exporte, Kredite, usw.) und konnten ganz legitim in bilaterale Kooperationsabkommen aufgenommen werden. Obwohl diese Abkommen von der EWG-Kommission strikt geprüft wurden und ihre Inhalte oft für Konflikte sorgten, setzte die DDR bei der Entwicklung ihres Westhandels voll und ganz auf diesen Rahmen. Ein favorisiertes Modell stellten kreditfinanzierte Kompensationsgeschäfte dar ${ }^{(17)}$. Die DDR importierte beispielsweise Industrieanlagen und konnte zumindest einen Teil des Importwerts in Waren zurückzahlen. Oft spielten metallurgische Produkte eine große Rolle. Der europäische Stahlsektor war aber in den 1970er-Jahren in eine immer tiefere Krise geraten. Wenig überraschend folgten in diesem Bereich

15 Sitzung des SED-Politbüros am 7.3.1975, SAPMO-BArch, DY 30/J IV 2/2A/1864, B1. 65-75. Siehe auch: J. Wüstenhagen, „Blick durch den Vorhang“ (Anm. 2), S. 284-285.

16 Sitzung des SED-Politbüros am 1. März 1977, SAPMO-BArch, DY 30/J IV 2/2A/2047. Für die Dokumentation der Verhandlungen siehe: Politisches Archiv des Auswärtigen Amts, Berlin, (PA/AA), Bestand Ministerium für Auswärtige Angelegenheiten der DDR (MfAA), M 1 C 7135, B1. 1-191.

17 Matthias JudT, „Kompensationsgeschäfte der DDR - Instrumente einer europäischen Ost-West-Wirtschaftsintegration?", Jahrbuch für Wirtschaftsgeschichte, 49/2 (2008), S. 117-138. 
ebenfalls Schritte der EWG, galt es doch östlichen Dumping-Lieferungen entgegenzuwirken und den eigenen Stahlsektor zu schützen.

Der Davignon-Plan und die Entscheidung des Europäischen Rats vom Dezember 1977, dass alle Staaten, die Stahl in den EWG-Markt exportierten, in Zukunft bilaterale Vereinbarungen mit der Gemeinschaft zu schließen hätten, traf die DDR merklich. Während die Tschechoslowakei, gefolgt von Rumänien, Polen und Ungarn Abkommen mit der EWG schlossen, blieben die Sowjetunion und die DDR auf ihrem Standpunkt, verweigerten sich Verhandlungen und hatten die Konsequenzen zu tragen. Ein hoher Prozentsatz der ostdeutschen Stahlexporte schien gefährdet $^{(18)}$. Die Realisierung der im Rahmen von Kompensationsgeschäften vereinbarten Warenlieferungen musste nun mühsam durchgesetzt werden. Trotz bilateraler Verhandlungserfolge (Italien) ${ }^{(19)}$ kam es zu signifikanten Exportausfällen (Großbritannien) ${ }^{(20)}$. Bald zeigte sich auch in anderen Bereichen (z. B. Textilwaren), dass neue Importkontingente der EWG-Staaten den DDR-Export beeinträchtigten - und dies in einer Zeit, als die DDR verzweifelt danach trachtete ihre Westexporte auszuweiten, um ihr Außenhandelsdefizit und die steigende Verschuldung in den Griff zu bekommen ${ }^{(21)}$. Anstatt wie andere sozialistische Staaten ökonomisch vorteilhafte Sektorenabkommen mit der EWG auszuhandeln, sah sich die DDR mit einer endlosen Serie von Anti-DumpingMaßnahmen gegen ihre Importe in den Gemeinsamen Markt konfrontiert ${ }^{(22)}$.

\section{Kurze Beziehungen mit langem Anlauf}

Der RGW hatte sich 1975 dazu entschlossen, direkte Verhandlungen mit der EWG zu beginnen. Im Endeffekt kam bei den sich in der Folge entwickelnden Gesprächskontakten nichts heraus, denn die EWG weigerte sich, den RGW als gleichgeartete Integrationsform anzuerkennen und auf dessen Forderung nach einem den Handel einschließenden Abkommen einzugehen. Rasch war man an einem toten Punkt angelangt. Anfang der 1980er-Jahre herrschte vollkommener Stillstand. 1984 setzte die Sowjetunion erneut Schritte zu einer besseren Koordination im RGW und zur Wiederaufnahme der seit 1980 unterbrochenen Kontakte zur EWG. Die DDR begrüßte das Vorhaben ${ }^{(23)}$, denn es war klar, „daß die Länder der EWG auch in Zukunft die

18 Information über die Auswirkungen der EWG-Maßnahmen auf metallurgischem Gebiet für die DDR, Berlin, 5.1.1978, SAPMO-BArch, DY 3023/1276, Bl.77-80.

19 Beil an Mittag, Berlin, 1.3.1979, SAPMO-BArch, DY 3023/1279, Bl.58-59.

20 Tautenhahn an Mittag, 21. September 1979, SAPMO-BArch, DY3023/1277, Bl.249-250.

21 Zur DDR-Wirtschaftsgeschichte und zur Entscheidungsfindung in der SED siehe: André STEINER, The Plans that Failed. An Economic History of the GDR, New York, Berghahn, 2010; Hans-Hermann Hertle, „Die Diskussion der ökonomischen Krisen in der Führungsspitze der SED“, in: Theo Pirker, M. Rainer Lepsius, Rainer Weinert, Hans-Hermann Hertle (Hg.), Der Plan als Befehl und Fiktion. Wirtschaftsführung in der DDR. Gespräche und Analysen, Opladen, Westdeutscher Verlag, 1995, S.309-345; Matthias JudT, Der Bereich Kommerzielle Koordinierung. Das DDR-Wirtschaftsimperium des Alexander Schalck-Golodkowski - Mythos und Realität, Berlin, Links, 2013; Andreas MALYCHA, Die SED in der Ära Honecker, Machtstrukturen, Entscheidungsmechanismen und Konfliktfelder in der Staatspartei 1971-1989, München, Oldenbourg Wissenschaftsverlag, 2014, S. 177-322.

22 Als Übersicht für den Zeitraum 1978-1981 siehe: Information Nr. 165/1981 über Antidumpingverfahren der EWG gegen Außenhandelsbetriebe der DDR, Berlin, 16.9.1981, BArch, DL 2/6380, B1. 296-298. 
wichtigsten Handelspartner der RGW-Länder unter den kapitalistischen Industrieländern bleiben“ würden. Zwar wurden weiterhin offizielle Beziehungen zwischen RGW und EWG angestrebt, aber man war sich bewusst, dass die EWG nicht bereit sein würde, „einem Abkommen zuzustimmen, in dem konkrete handelspolitische Fragen zwischen beiden Organisationen geregelt werden “(24). Unabhängig von der weiteren Entwicklung, blieb das unveränderte Bestehenbleiben des innerdeutschen Handels für die DDR von zentraler Bedeutung ${ }^{(25)}$.

Unter Gorbatschow vollzog sich ab Frühjahr 1985 eine massive Änderung der sowjetischen Haltung zur EWG. Nach Sondierungskontakten erfolgte schließlich im September 1986 die Wiederaufnahme der Verhandlungen zwischen RGW und EWG ${ }^{(26)}$. Die Grundsatzabteilung des DDR-Außenministeriums hielt nun eindeutig fest, dass die „bisherigen Erfahrungen“ zeigten, „daß ein weiteres Zögern bei der Herstellung offizieller Beziehungen RGW-EWG unsere Verhandlungspositionen nicht verbessert“. In den vergangenen Jahren war es der EWG immer mehr "gelungen, unter Ausnutzung konkreter ökonomischer Interessen der RGW-Länder diese dazu zu drängen in verschiedenen Sachbereichen und auf verschiedenen Ebenen technische und teilweise darüber hinausgehende Kontakte mit der EWG-Kommission aufzunehmen“. Diese weiterhin als „Differenzierungspolitik“ bezeichnete Entwicklung wurde nun „sowohl aufgrund bereits bestehender bilateraler Vereinbarungen der EWG mit den meisten RGW-Ländern als auch angesichts des zunehmenden ökonomischen Drucks seitens der EWG“ als unumkehrbar angesehen. Daher erachtete man es als erforderlich, „früher oder später die Phase ,vertragsloser' Beziehungen auf dem Gebiet des Handels zu beenden“. Zudem wurde die EWG langfristig auch als Partner für den politischen Dialog der DDR gesehen. Den Anfang sollten parlamentarische Kontakte machen ${ }^{(27)}$.

Bereits im Juni 1986 erfolgte der Besuch einer Delegation der Fraktion sozialistischer Parteien im Europäischen Parlament in Ost-Berlin. Volkskammerpräsident Horst Sindermann bekundete im Oktober 1986 die ostdeutsche Bereitschaft zur Aufnahme von Parlamentsbeziehungen. In weiterer Folge nahm der Delegationsaustausch Fahrt auf. Den Höhepunkt stellte aus DDR-Sicht der Besuch Sindermanns im Europäischen Parlament in Strasbourg vom 12. bis 14. Oktober 1988 dar, der insbesondere zum Austausch über Abrüstungs- und Sicherheitsfragen genutzt werden sollte. Sindermann kehrte nach zwei Tagen intensiver Diskussion zufrieden in die DDR zurück und berichtete von einer „aufgeschlossenen und konstruktiven Atmosphäre“. Unangenehmes war ihm aber freilich nicht erspart geblieben, wie sein Bericht vermerkte:

„Es wurden Fragen zur Haltung der DDR zum Prozeß der Umgestaltung in der Sowjetunion gestellt und zur Entwicklung in einigen Ländern des Sozialismus, wie Ungarn und Polen. Vor allem von Vertretern der Fraktion der Europäischen Volkspartei wurde von der

24 Sitzung des SED-Politbüros am 16.10.1984, SAPMO-BArch, DY 30/J IV 2/2A/ 2696, Bl. 176-182.

25 Zur Vorlage „Standpunkt der DDR zum weiteren Vorgehen gegenüber den Europäischen Gemeinschaften“, Schalck an Mittag, Berlin, 14.2.1985, SAPMO-BArch, DY 3023/1315, B1.277-279.

26 Wolfgang Mueller, „Die UdSSR und die europäische Integration“, in: Michael Gehler (Hg.), Vom Gemeinsamen Markt zur Europäischen Unionsbildung. 50 Jahre Römische Verträge 1957-2007, Wien/ Köln/Weimar, Böhlau, 2009, S. 617-662, hier S. 659.

27 HA Grundsatzfragen und Planung. Zu außenpolitischen Fragen der Aufnahme offizieller Beziehungen RGW-EWG, Berlin, 20.3.1986, PA/AA, MfAA, ZR 3206/13. 
DDR gefordert, die ,Mauer in Berlin zu beseitigen. Diese Leistung müßte von der DDR erbracht werden als Voraussetzung für mehr Vertrauen in Europa. Darüber gab es zum Teil heftige Angriffe auf die Politik der DDR, bis zu einzelnen Provokationen von rechten Politikern“"(28).

Das SED-Politbüro wollte den Besuchsaustauch dennoch weiter intensivieren ${ }^{(29)}$. Nachdem Außenminister Oskar Fischer entsprechend den Direktiven des SED-Politbüros in einem Schreiben an den Kommissar für Handel und Außenpolitik Willy de Clercq am 28. Mai 1986 die Bereitschaft der DDR zur Aufnahme von offiziellen Beziehungen signalisiert hatte, kam es im November 1986 zu ersten Expertengesprächen. In diesen betonte die DDR den für sie bestehenden Zusammenhang zwischen ihrem Verhältnis zu der EWG und der Normalisierung der Beziehungen zwischen RGW und EWG sowie ihr Bestehen auf den Fortbestand des innerdeutschen Handels. Die EWG strebte „kurzfristig kein Handelsabkommen“ mit der DDR an, was aber die Lösung anstehender "Sachfragen auf dem Verhandlungswege“ nicht ausschloss ${ }^{(30)}$. Ganz in diesem Sinne wurde im November 1987 ein Sektoralabkommen über den Handel mit Schafen und Ziegen sowie Schaf- und Ziegenfleisch geschlossen, das der DDR einen jährlichen Export von Schlachtschafen in die EWG im Wert von 20 Millionen Valutamark sicherte ${ }^{(31)}$. Zur Fortsetzung der Expertengespräche kam es erst im Juni $1988^{(32)}$. Nach der Herstellung offizieller Beziehungen zwischen dem RGW und der EWG am 25. Juni 1988 nahm die DDR schließlich am 10. August 1988 offizielle Beziehungen zur EWG auf und etablierte im Februar 1989 in Brüssel eine Mission der DDR bei der EWG.

Im Zentrum des ostdeutschen Interesses stand nun die Aufnahme von Handelsvertragsverhandlungen, zu denen sich Brüssel im Sommer 1988 grundsätzlich bereit erklärt hatte. Der Grund dafür lag auf der Hand: Auch die DDR konnte sich den Konsequenzen der Schaffung eines EWG-Binnenmarkts bis 1992 nicht entziehen. Unter Einbeziehungen des Handels mit der Bundesrepublik entfielen 80\% des Westhandelsumsatzes der DDR auf diesen Raum. Es war klar, dass der Binnenmarkt zu einer Verschärfung der Absatz- und Konkurrenzbedingungen würde, jedoch hoffte man bei entsprechender Vorbereitung auch auf neue Absatzchancen. Daher hatte sich das SED-Politbüro im September 1988 grundsätzlich zum Abschluss eines Handelsabkommens „bei Aufrechterhaltung des bestehenden Systems des Handels DDR-BRD“ entschlossen. Ost-Berlin erwartete, dass die EWG bereit sei, „den Prozeß der Liberalisierung im Abkommen zu fixieren“, jedoch „ohne einen Endtermin für die völlige Aufhebung der Mengenbeschränkungen festzusetzen“ und die „Gewährung der Meistbegünstigung von effektiven Gegenleistungen abhängig“ zu machen. Darunter fielen der erleichterte Zugang zum DDR-Markt und Kontakte mit Endkunden, was

28 Information über den Besuch einer Delegation der Volkskammer der DDR unter Leitung ihres Präsidenten Horst Sindermann im Europäischen Parlament vom 12. bis 14.10.1988, PA/AA, MfAA, ZR2655/95.

29 Sitzung des SED Politbüros am 25.10.1988, SAPMO-BArch, DY 30/J IV 2/2/2300, Bl. 81.

30 Bericht über die Expertengespräche DDR-EG am 12. und 13.11.1986 in Brüssel, PA/AA, MfAA, ZR 3206/13.

31 Information, gez. Beil, Berlin, 7.12.1987, SAPMO-BArch, DY 3023/1297, B1. 321.

32 Bericht über die Expertengespräche DDR-EG am 27. und 28.6.1988 in Brüssel, ZR 2657/95. 
einer weitgehenden Öffnung gleichkam. Zur Wahrung der Exportinteressen ergab sich die „Zwingende Notwendigkeit“ interner Anpassungen und Veränderungen, um „den komplizierten Bedingungen und steigenden Anforderungen des EWG-Binnenmarktes“ (hinsichtlich Standards und Richtlinien der Qualitätssicherung, des Konsumentenschutzes und der Produkthaftung) gerecht zu werden ${ }^{(33)}$.

Die Bundesrepublik informierte die DDR detailliert über die zu erwartenden Auswirkungen des Binnenmarkts auf den innerdeutschen Handel. Formal wurde dieser durch die Vertiefung der Integration zwar nicht berührt, die Verpflichtung Bonns Störungen des Gemeinsamen Markts resultierend aus dem Handel zwischen den beiden deutschen Staaten „zu vermeiden“ war aber aktueller denn je. Anfang 1989 betrug das Re-exportvolumen von DDR-Waren, die über die Bundesrepublik in die übrigen EWG-Staaten verbracht wurden ca. 50 Millionen DM und somit lediglich 0,02\% des Warenexports der DDR. Bonn erwartete dennoch, „daß der Druck der EWG-Partner auf die BRD“ zunehmen würde, „um abzusichern, daß die im Rahmen des Handels mit der BRD verbrachten DDR-Waren auch in der BRD verbleiben“(34).

Nicht zuletzt deshalb zögerte die EWG-Kommission auch nach erfolgreichem Abschluss der Sondierungsgespräche mit der DDR - bei denen eine Nichtberührungsklausel zum innerdeutschen Handel vereinbart worden war - das für den Beginn der Abkommensverhandlungen erforderliche Mandat zu beantragen. Um die Zurückhaltung Brüssels zu überwinden, lud die DDR die verantwortlichen EWG-Kommissare Frans Andriessen und Martin Bangemann sowie den EWG-Parlamentspräsidenten Barón Crespo zum Besuch nach Ost-Berlin ein ${ }^{(35)}$.

Der Augenblick schien ungünstig, da sich die innere Lage der DDR im Verlauf des Jahres 1989 sukzessive zugespitzt hatte. Nach Protesten gegen die gefälschten Ergebnisse der Kommunalwahlen im Mai, hatte sich in den Sommermonaten die Fluchtbewegung ausgeweitet und mit der Grenzöffnung durch Ungarn am 10. September endgültig Bahn gebrochen. Die „friedliche Revolution“ nahm Fahrt auf und am 17./18. Oktober wurde Erich Honecker zum Rücktritt veranlasst. Sein Nachfolger Egon Krenz verkündete eine „Wende“, Anfang November war die weitere Entwicklung aber noch gänzlich unabsehbar ${ }^{(36)}$.

Massenprotest und verzweifelte Reformbemühungen prägten das Tagesgeschehen, als der Vizepräsident der EWG-Kommission Bangemann in seiner Funktion als Kommissar für Binnenmarkt und Industriepolitik Anfang November in die DDR kam. Dort stellte er gleich zu Beginn vor Medienvertretern fest, „daß sein Besuch in der DDR, der ursprünglich rein fachlich auf eine Diskussion von beiderseits interessierenden Fragen des Binnenmarktes der EWG orientiert war, durch die Entwicklung eine neue politische Dimension erhalten habe“. DDR-Außenminister Oskar Fischer versicherte ihm die Ernsthaftigkeit der „Wende“ und gab sich überzeugt: „Mit der Wende werde die DDR zugleich noch attraktiverer Partner sein.“ Gleichzeitig betonte er, „daß das

33 Sitzung des SED-Politbüros am 21.3.1989, SAPMO-BArch, DY 30/J IV 2/2A/3204, Bl. 80-109.

34 Vermerk über ein Gespräch zu Fragen der Herausbildung des EG-Binnenmarktes anläßlich der Verhandlung mit der Treuhandstelle für Industrie und Handel am 15.2.1989, SAPMO-BArch, DY 3023/1337, B1.402-404. 
Aufwerfen der Frage der,Wiedervereinigung ' müßig sei und die realistische Ost-WestZusammenarbeit nur stören könne“. Bangemann erklärte, „daß er in der ,Wende in der DDR` eine Erleichterung für den Bau des Hauses Europa sehe. Die Diskussionen, daß sich die BRD zwischen EWG-Integration und Wiedervereinigung entscheiden müsse“, hielt er „für unrealistisch“. Als er auf das Bonner Grundgesetz Bezug nahm, unterbrach ihn Fischer „mit dem Hinweis, daß er diese Argumente alle schon von Genscher gehört habe“. Bangemann betonte daraufhin einerseits die potentielle politische Brisanz des Themas in der Bundesrepublik, andererseits hielt er auch fest: „Es gebe 2 deutsche Staaten, und die DDR und die UdSSR wollten, daß das sozialistische Deutschland bestehen

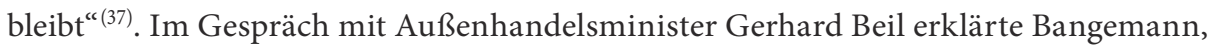
dass die EWG und ihre Mitgliedsländer „, an einer effizienten DDR-Wirtschaft interessiert“ seien und in den sozialistischen Ländern „ein echtes Potential für die Erweiterung der Außenwirtschaftsbeziehungen der EWG“ sehen würden ${ }^{(38)}$.

Abschließend traf Bangemann mit dem neuen Staats- und Parteichef Egon Krenz zusammen. Ihm gegenüber bemerkte Bangemann, „daß man jetzt im Westen sehr auf die Taten sehe, die in der DDR ergriffen werden“. Mit Blick auf die Beziehungen der DDR zur EWG führte er aus: „Die beiden deutschen Staaten unterhielten in gewissem Sinne privilegierte, besondere Beziehungen, die man immer wieder in den allgemeinen Rahmen einpassen müsse, denn sie seien für andere Staaten (der EWG) insofern immer kompliziert.“ Krenz gab seiner Hoffnung Ausdruck, dass der Handelsvertrag bald vorankommen würde, erklärte für Kooperation offen zu sein und betonte abschließend die Souveränität der DDR ${ }^{(39)}$. Das DDR-Außenministerium betonte in seiner internen Einschätzung das „politische Gewicht“ des Besuchs, gegen den „es in der EWG-Kommission starke Einwände“ gegeben hätte. Im Gegensatz zu Bangemann war der für die Außenbeziehungen und ein Handelsabkommen zuständige EWGKommissar Andriessen „noch nicht bereit, der an ihn ergangenen Einladung Folge $\mathrm{zu}$ leisten ${ }^{\text {( }}(40)$.

Unterdessen ging der Kollaps des SED-Regimes in seine nächste Phase. Am 9. November erfolgte die in dieser Form unbeabsichtigte Öffnung der Berliner Mauer, wenige Tage später wurde eine neue Regierung unter der Leitung von Hans Modrow gebildet. Diese hatte trotz aller inneren Turbulenzen am 17. November in einem Memorandum ihre Vorstellungen zur Zusammenarbeit mit der EWG dargelegt und eine positive Antwort des amtierenden EWG-Ratsvorsitzenden François Mitterrand erhalten. Am 28. November präsentierte Bundeskanzler Helmut Kohl im Deutschen Bundestag jedoch bereits sein Zehn-Punkte-Programm, das einen Fahrplan für einen über Zwischenschritte zu erreichenden Weg zur deutschen Einheit entwarf ${ }^{(41)}$.

37 Vermerk über das Gespräch Fischer - Bangemann am 1.11.1989, PA/AA, MfAA, ZR 2661/95.

38 Vermerk über das Gespräch Beil - Bangemann, Berlin, 2.11.1989, PA/AA, MfAA, ZR 2661/95.

39 Vermerk über das Gespräch Krenz - Bangemann am 2.11.1989, PA/AA, MfAA, ZR 2661/95.

40 Besuch des Vizepräsidenten der EG-Kommission, Dr. Martin Bangemann, in der DDR, PA/AA, MfAA, ZR 2661/95.

41 Michael GeHLer, „Von der Befürwortung zur Verzögerung und Verhinderung: Österreichs EG-Antragsgesuch, die Bundesrepublik und die Annäherungen der DDR an die Europäischen Gemeinschaften 1989-1990“, in: Ders., Maximilian Graf (Hg.), Europa und die deutsche Einheit. Beobachtungen, Entscheidungen und Folgen, Göttingen, Vandenhoeck \& Ruprecht, 2017, S. 295-348, hier S. 310-314. 
Nach einer Expertenberatung mit der EWG-Kommission am 1. Dezember in Brüssel, in der es um ein besseres Kennenlernen der ostdeutschen Standpunkte ging, hatte man in Ost-Berlin den Eindruck, dass die „gesellschaftlichen Veränderungen in der DDR [...] zu einer wesentlichen Veränderung der EWG-Position geführt" hätten und „die EWG nunmehr an einem schnellen Abschluß eines Handels- und Wirtschaftsabkommens mit der DDR interessiert" $\operatorname{sei}^{(42)}$.

Als Frans Andriessen wenige Tage später die DDR besuchte, zeigte er im Gespräch mit der neuen DDR-Wirtschaftsministerin Christa Luft „starkes Interesse [...] an den Grundlinien der politischen Reformen, insbesondere der Wirtschaftsreformen “(43). Umweltschutzminister Hans Reichelt informierte den EWG-Kommissar über die gestiegene Bedeutung der Agenden seines Ressorts ${ }^{(44)}$. Außenminister Fischer betonte in gewohnter Manier, dass man bei der Zusammenarbeit in Europa von den Realitäten und somit der „Existenz zweier deutscher Staaten“ auszugehen habe. Andriessen ging auf die deutsche Frage folgendermaßen ein:

„Die Beziehungen der DDR zur BRD, und wie sie weiter ausgestaltet werden, seien für die EWG wichtig. Dabei achten sie besonders auf zwei Elemente; die Souveränität beider Staaten und ihren Platz in Europa. Dies seien Realitäten, die man beachten muß. Darauf basierend entsteht aus den Beziehungen DDR-BRD auch mehr Platz für die Kooperation DDR-EWG“(45).

Gegenüber Ministerpräsident Modrow bezeichnete Andriessen seine Gespräche für die Erarbeitung der Begründung zum weiteren Vorgehen gegenüber der DDR, die er der EG-Kommission am 6. Dezember vorlegen musste, als „nützlich“. Der ostdeutsche Regierungschef informierte den EWG-Kommissar über die jüngsten Entwicklungen in der DDR. Vor dem Hintergrund der Veröffentlichung eines Appells Kulturschaffender unter dem Motto „Für unser Land“ gab er sich überzeugt: „Wir bleiben ein souveräner deutscher Staat mit einem sich erneuernden Sozialismus.“ Dementsprechend kündigte er an bei seinem Treffen mit Bundeskanzler Kohl am 19. Dezember in Dresden davon auszugehen, dass dieser im vierten Punkt seines Zehn-Punkte-Plans den „Vorschlag zur Schaffung einer Vertragsgemeinschaft beider deutscher Staaten akzeptiert habe“. Jedoch betonte er: „Eine ,Wiedervereinigung beider souveräner deutscher Staaten, so wie sie in Kohls Erklärung gestellt sei, komm[e] nicht in Frage." Für Modrow war diese Haltung auch „höchstes Staatsinteresse“ Frankreichs und Polens - eine Sichtweise, die sogar in seinem eigenen Außenministerium handschriftlich mit einem Fragezeichen versehen wurde. Weiters führte er aus: „Die DDR sei aufgeschlossen, die Beziehungen zur BRD ausgehend von der Existenz zweier souveräner deutscher Staaten zu entwickeln. Das gelte ebenso für die Beziehungen zu allen EWG-Staaten. Wir wollen keine Einbahnstraße zur BRD.“ Andriessen gab zu verstehen, dass er davon ausging, „daß die Zeit reif sei, die Zusammenarbeit zwischen der EWG und der DDR

42 Information über die Beratung mit der EG-Kommission [Brüssel, 1.12.1989], PA/AA, MfAA, ZR2728/13.

43 Vermerk über das Gespräch Luft - Andriessen am 4.12. in Berlin, PA/AA, MfAA, ZR 2728/13.

44 Vermerk über das Gespräch von Reichelt mit einer Delegation der EG-Kommission unter der Leitung von Andriessen am 5.12.1989 in Berlin, PA/AA, MfAA, ZR 2728/13.

45 Vermerk über das Gespräch Fischer - Andriessen, PA/AA, MfAA, ZR 2728/13. 
zügig zu entwickeln“. Seine weiteren Ausführungen dürften Balsam auf Modrows Seele gewesen sein:

„Er habe verstanden, daß der Prozeß der Demokratisierung in der DDR unumkehrbar ist. Das sehe er als Basis für die Entwicklung der weiteren Zusammenarbeit der EG mit der DDR an.

Zur Frage einer Vertragsgemeinschaft DDR-BRD, die im ,Kohl-Plan` nur eine Stufe sei, möchte er zwei Dinge sagen: Die EWG habe ein Interesse daran, daß die Entwicklungen in Mitteleuropa erstens von den bestehenden politischen Realitäten (Grenzen, 2 souveräne deutsche Staaten) ausgehen und zweitens, daß sie eingebettet in eine europäische Konzeption verlaufen “(46).

Das Treffen des Europäischen Rats in Strasbourg am 8./9. Dezember 1989 stand im Schatten multipler Widerstände gegen eine mögliche deutsche Wiedervereinigung. Dennoch wurde die deutsche Einheit als politisches Ziel der EWG keineswegs aufgegeben $^{(47)}$. Kurz vor Weihnachten sprach sich der EWG-Außenministerrat für Verhandlungen über ein Handels- und Kooperationsabkommen mit der DDR aus und die Wirtschaftsminister der Gemeinschaft genehmigten auf ihrem Treffen das Verhandlungsmandat der Kommission. Anfang 1990 setzte eine rege Beschäftigung der EWG-Kommission mit dem künftigen Verhältnis zur DDR ein. Drei Arbeitsgruppen wurden eingesetzt. Bald zeigte sich eine immer offenere Unterstützung der Kommission für die deutsche Einheit. Die Übergangsregierung unter Modrow setzte in ihrem Kampf um den Erhalt der Eigenstaatlichkeit der DDR dennoch einige Hoffnung in die EWG und strebte kurz vor ihrer Abwahl am 18. März 1990 sogar eine eigenständige Mitgliedschaft an. Zu diesem Zeitpunkt waren die im Januar aufgenommenen Verhandlungen über das Handelsabkommen bereits abgeschlossen. Kanzler Kohl bremste auf dem Weg zu seiner Unterzeichnung am 8. Mai und das Dokument erlangte aufgrund des voranschreitenden Einigungsprozesses schließlich keine Gesetzeskraft mehr ${ }^{(48)}$. Unter der frei gewählten Regierung Lothar de Maizière ging es dann vor allem darum, das Gebiet der DDR möglichst rasch EWG-fit und -kompatibel zu machen, ehe dieses mit Vollzug der deutschen Einheit am 3. Oktober 1990 zu einem Teil der Gemeinschaft wurde ${ }^{(49)}$.

\section{Schlussbetrachtung: deutsche und europäische Einheit}

Die DDR war unter den sozialistischen Staaten beim Umgang mit der EWG in jedweder Hinsicht ein Sonderfall und Spätstarter, ironischerweise wurde ihr Territorium durch „friedliche Revolution“ und rasche Vereinigung vor allen Transformationsstaaten Teil des geeinten Europas. Die Gründe dafür lagen in der deutschen Teilung, dem bundesdeutschen Alleinvertretungsanspruch und dessen zumindest formal

46 Vermerk über das Gespräch Modrow - Andriessen am 5.12.1989, PA/AA, MfAA, ZR 2728/13.

47 „Wir streben die Stärkung des Zustands des Friedens in Europa an, in dem das deutsche Volk in freier Selbstbestimmung seine Einheit wiedererlangt." Zit. nach: Schlußfolgerungen des Europäischen Rates der Staats- und Regierungschefs zur Tagung vom 8.-9.12.1989, Europa-Archiv, 1 (1990), S. D 14.

48 M. Gehler, „Von der Befürwortung zur Verzögerung und Verhinderung“ (Anm. 41), S. 315-329.

49 Carsten Meyer, Die Eingliederung der DDR in die EG, Köln, Verlag Wissenschaft und Politik, 1993; Beate Kohler-Koсн (Hg.), Die Osterweiterung der EG. Die Einziehung der ehemaligen DDR in die Gemeinschaft, Baden-Baden, Nomos, 1991. 
durchgängiger Akzeptanz im Westen. Während sich die Eingliederung der DDR in die EWG durch die Vereinigung mit der Bundesrepublik im Endeffekt schließlich ganz gemäß den seit 1957 von der Gemeinschaft vertretenen politischen Prämissen vollzog, so waren die Beziehungen Ost-Berlins zum Gemeinsamen Markt insbesondere in den 1970er- und 1980er-Jahren komplexer als gemeinhin angenommen. Trotz des aus dem innerdeutschen Handel resultierenden vorteilhaften Sonderstatus war die DDR im Verhältnis zum Rest der EWG von den ökonomischen Auswirkungen der fortschreitenden westeuropäischen Integration im gleichen Ausmaß wie die anderen RGW-Staaten betroffen. Dennoch verfolgte Ost-Berlin bis Mitte der 1980er-Jahre die unflexible sowjetische Linie der Nichtanerkennung der EWG, die aber spätestens ab 1975 nicht konsequent durchgehalten werden konnte. Trotz des Primats einer unveränderten innerdeutschen Handelspraxis konnte und wollte sich die DDR den Auswirkungen des aufziehenden EWG-Binnenmarkts nicht entziehen. Im Gegenteil, die EWG sollte nun nicht nur als ökonomischer Faktor, sondern auch als politischer Partner gesehen werden. Das rasche Ende der kurzen Beziehungen mit langer Anlaufzeit kam aber jäh. Die Aussagen einiger EWG-Vertreter im Herbst dürften die Illusion bestärkt haben, dass Beziehungen mit Brüssel den Fortbestand der Eigenstaatlichkeit der DDR stützen könnten. Tatsächlich war die EWG im Gegensatz zu einigen ihrer Mitgliedsstaaten von Anfang an - wie Piers Ludlow festgehalten hat - ein „naturally supportive environment" für die deutsche Einheit ${ }^{(50)}$.

\section{Zusammenfassung}

Der Artikel behandelt das Verhältnis der DDR zur EWG von 1957 bis 1990. Ausgehend von einer problemorientierten Einführung in die deutsch-deutschen und europäischen Rahmenbedingungen wird die politische und ökonomische Dimension der Beziehungen in den Blick genommen. Dabei wird deutlich, dass die DDR trotz ihres aus dem innerdeutschen Handel resultierenden Sonderstatus wie alle anderen RGW-Staaten von den Auswirkungen der sich vertiefenden westeuropäischen Integration betroffen war. Dennoch weigerte sich Ost-Berlin der Moskauer Linie folgend die EWG anzuerkennen. Erst Mitte der 1980er-Jahre intensivierten sich die Kontakte und 1988 wurden Beziehungen etabliert. Obwohl die sich entwickelnde Europäische Gemeinschaft zumindest formal stets für eine Wiedervereinigung Deutschlands eintrat, hoffte die DDR-Führung auf Brüsseler Unterstützung in ihrem Kampf um den Erhalt ihrer Eigenstaatlichkeit.

\footnotetext{
Abstract

The article deals with the relations between the GDR and the EEC from 1957 to 1990. After a problem-oriented introduction to the German-German and European framework of this relationship, it focusses on its political and economic dimension. In doing

50 N. Piers Ludlow, „A naturally supportive environment? The European institutions and German unification“, in: Frédéric Bozo, Marie-Pierre Rey, N. Piers Ludlow, Leopoldo Nuti (Hg.), Europe and the End of the Cold War. A reappraisal, London, Routledge, 2008, S. 161-173; N. Piers LudLow, „Not a wholly new Europe: how the integration framework shaped the end of the Cold War in Europe", in: Frédéric Bozo, Andreas Rödder, Mary Elise Sarotte (Hg.), German Reunification. A multinational history, London, Routledge, 2017, S. 133-152.
} 
so, the analysis shows that - despite the special status resulting from the provisions of intra-German trade - the GDR was no less affected by the progress of deepening West European integration than the other COMECON member states. Nevertheless, following Moscow's line, East Berlin refused to officially recognize the EEC. Only in the mid1980 s contacts intensified and relations were established in 1988. Despite the fact, that the developing European Community had - at least formally - always supported the West German goal of reunification, ironically, the GDR leadership hoped for support by Brussels in its struggle for the survival of its state.

\section{Résumé}

L'article traite des relations entre la RDA et la CEE de 1957 à 1990. Après une introduction consacrée aux contextes germano-allemand et européen dans lesquels s'inscrivent ces relations, leur dimension politique et économique est examinée. Il apparaît clairement que, malgré son statut spécial résultant du commerce intra-allemand, la RDA, comme tous les autres pays du CAEM, a été affectée par la construction de l'Europe occidentale. Suivant la ligne de Moscou, Berlin-Est refusait néanmoins de reconnaître la CEE. Ce n'est qu'au milieu des années 1980 que les contacts se sont intensifiés et qu'en 1988, des relations ont été établies. Bien que la Communauté européenne ait toujours formellement préconisé la réunification de l'Allemagne, les dirigeants de la RDA espéraient que Bruxelles soutiendrait leur lutte pour préserver leur État. 